\title{
Impact of SARS-CoV-2 on Cardio-Pulmonary-Immune Signatures: A Review of Recent Developments with a Look toward the Future
}

Charan Reddy Kudumula

Department of Clinical and Interventional Cardiology, Lilavati Hospital and Research Center, Mumbai, India.

Corresponding author: Charan Reddy Kudumula, Department of Clinical and Interventional Cardiology, Lilavati Hospital and Research Center, Mumbai, India.

Received date: September 30, 2020; Accepted date: October 12, 2020; published date: October 23, 2020

Citation: Charan R. Kudumula. Impact of SARS-CoV-2 on Cardio-Pulmonary-Immune Signatures: A Review of Recent Developments with a Look toward the Future. J Clinical Research Notes, 1(4); DOI:10.31579/2690-8816/028

Copyright: (c) 2020 Charan Reddy Kudumula. This is an open access article distributed under the Creative Commons Attribution License, which permits unrestricted use, distribution, and reproduction in any medium, provided the original work is properly cited.

\begin{abstract}
In December 2019, the first case of patient with severe acute respiratory syndrome (SARS) caused by the novel coronavirus, SARS-CoV-2, was reported in Wuhan, Hubei Province, Central China. SARS-CoV-2 causes CoronaVirus Disease-2019 (COVID-19 or nCoV-19), which rapidly spread all over the world. In response to this serious situation, on 30 January 2020, the World Health Organization (WHO) declared a global public health emergency of international concern, putting all health organizations on high alert. In mid-March 2020, WHO declared nCoV-19 a pandemic. By the end of September 2020, there were $~ 33.67$ million confirmed cases and $\sim 10.08$ lakh deaths worldwide and the trend rising on each passing day. SARS-CoV-2 mainly infects the pulmonary system, but cause damage to other organs such as heart, kidney and intestine. Understanding the cardio-pulmonary issues underpinning of nCOV-19 pathogenesis is key to managing outcomes and mortality. SARS-CoV-2 uses the SARS-CoV receptor, ACE2 for entry and the serine protease inhibitor TMPRSS2 for S protein priming. Alveolar cells and cardiomyocytes express ACE2 and TMPRSS2. In this Review, we summarize the current understanding of nCOV-19 pandemic from basic mechanisms to clinical perspectives, focusing on the interaction between SARS-CoV-2 and the cardio-pulmonary-immune signatures. The study provides crucial insights into the first step of SARS-CoV-2 infection, and potential targets for antiviral intervention.
\end{abstract}

Keywords: novel coronavirus; SARS-CoV-2; n CoV-2019, immunity; severe acute respiratory syndrome; middle east respiratory syndrome

\section{Introduction}

\section{History of SARS-CoV-2}

Coronaviruses (CoVs) have been identified as human pathogens since early 1960. CoVs belong to the subfamily Orthocoronavirinae in the family Coronaviridae, order Nidovirales. There are four genera within the subfamily Orthocoronavirinae, namely $\alpha$-coronavirus $(\alpha-\mathrm{CoV}), \beta$ coronavirus $(\beta-\mathrm{CoV}), \gamma$-coronavirus $(\gamma-\mathrm{CoV})$ and $\delta$-coronavirus $(\delta-\mathrm{CoV})$ [1]. Both $\alpha$-and $\beta$-CoV genera are known to infect mammals, whilst $\delta$-and $\gamma$-CoVs infect birds [2].

In the 21 st century, two highly infectious $\beta-\mathrm{CoVs}$, such as severe acute respiratory syndrome-coronavirus (SARS-CoV) was first reported in Guangdong province, China in 2002; and Middle East respiratory syndrome-coronavirus (MERS-CoV) first emerged in Saudi Arabia in 2012, subsequently spread to 32 countries [3-6]. SARS-CoV was mainly found in natural reservoir host, horseshoe bats (Rhinolophus affinis) and cause disease outbreak [7, 8]. Human transmission for SARS-CoV was facilitated by intermediate host like palm civet cats (Paguma larvata) and raccoon dogs (Nyctereutes procyonoides), which are frequently sold as food sources in Huanan seafood market, China [9, 10]. For MERS-CoV dromedary camels (Camelus dromedarius) are the reservoir host for human infections.

Etiology of nCoV-19 pandemic
In December 2019, a number of patients with pneumonia of unknown etiology emerged in Wuhan City, Hubei Province, Central China. Based on phylogeny, taxonomy, genome sequencing, and established practices of viruses, on February $11^{\text {th }}, 2020$, the Coronavirus Study Group (CSG) of international committee designated this novel virus as severe acute respiratory syndrome coronavirus-2 (SARS-CoV-2) previously known as 2019 novel coronavirus [11]. On February 12, 2020, World Health Organization (WHO) named the SARS-CoV-2 infection as a novel coronavirus disease-2019 (novel COVID-19) or nCoV-19. Considering the global threat, on January 30, 2020 the WHO has declared nCoV-19 pandemic a public health emergency of international concern (PHEIC).

It has been nine months since health officials reported spread of mysterious invisible SARS-CoV-2 infection in China. Infection quickly spread over 213 countries and territories around the world and 2 international conveyances. The nCOV-19 pandemic is changing our lives in unprecedented ways. At the time of publication of this article (end of September, 2020), based on the WHO epidemiological situation report [12], globally nCoV-19 pandemic has led to $~ 33.67$ million cases and $\sim 10.08$ lakh deaths. Since there are no clinically approved safe and effective vaccines or specific therapeutic anti-viral options available for nCOV-19 treatment, most authorities implemented few unprecedented measures to combat the viral transmission. These include the suspension of public transportation, locked down, mask wearing and social distancing. 


\section{Genome structure of SARS-CoV-2}

The SARS-CoV-2 is an enveloped, positive-sense, single-stranded RNA genome named for its solar corona like appearance [13]. Their helical symmetry nucleocapsid is $26-32 \mathrm{~kb}$ in size, making it the largest investigated genome among RNA viruses [14]. Like other CoVs, SARSCoV-2 particles are spherical and have 9-12 nm long special glycoproteins called a "spike" (peplomer) protruding from their surface. The outside of the viral particle is comprised of four main proteins: spike (S), envelope (E), membrane (M), and hemagglutinin esterase (HE) (Fig 1). Inside the viral particle, the viral RNA is encased in a shell made of the nucleocapsid $(\mathrm{N})$ protein. Notably, there are also 16 nonstructural proteins and 5-8 accessory proteins.

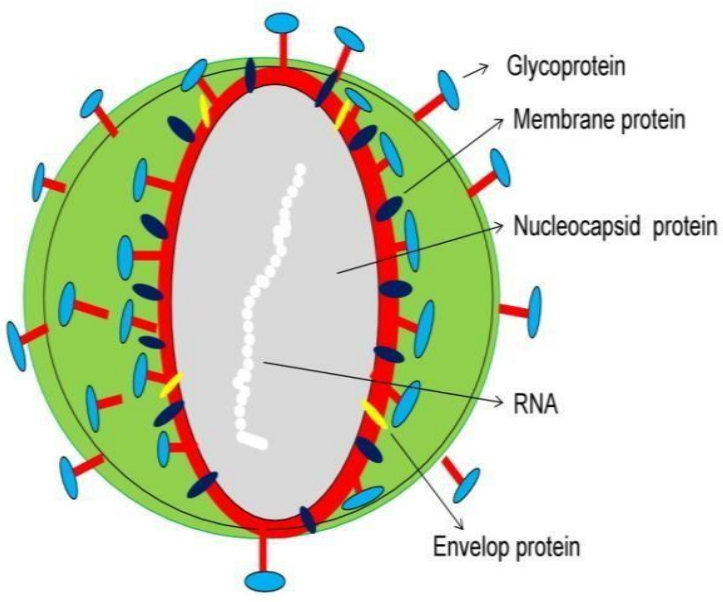

Fig.1. Structural organization of $\mathrm{nCoV}-19$.

Spherical envelop studded with club-shaped glycoprotein projections. Core matrix consists of membrane protein, nucleocapsid protein and envelope protein enclosed within which is a single-stranded RNA.The glycoproteins are responsible for attachment to the host cell and carry antigenic epitopes recognized by neutralizing antibodies.

SARS-CoV-2, the virus that causes nCOV -19 diseases, shares many biological features with SARS-CoV. A full-length genome sequence SARS-CoV-2 share $80 \%$ nucleotides (nt) sequence identity to SARS$\mathrm{CoVs}$ and $\sim 50 \%$ to MERS-CoVs. The SARS-CoV-2 sequence is $96 \%$ identical at the whole-genome level to a horseshoe bat coronavirus isolate RaTG13 and $88 \%$ identical to two bat-derived SARS-CoVs (bat-SLCoVZC45 and bat-SL-CoVZXC21) [15].

Recent studies have shown that like $\alpha$-CoVs, the spikes of SARS-CoV-2 latch onto cell surface, then undergo a structural modification that mediates fusion between the envelope and host cell membranes[16,17] via a metallopeptidase like angiotensin-converting enzyme-2 (ACE2) thereby produce more copies of viral particles [18]. SARS-CoV-2 primarily infects ciliated bronchial epithelial cells, type II pneumocytes and cardiomyocytes [15]. Therefore, the presence of such receptors and S protein primer proteases in these cells is a key determinant of SARS-CoV2 infection.

Human SARS-CoV-2 has a unique furin-like cleavage site, "RRAR motif" in the spike protein, which is not found in CoVs isolated from pangolins, indicating this virus may not have originated directly from pangolins [19]. The ACE2 abundantly express furin as well as endosomal protease, cathepsin-B (CTS-B). The main host protease that mediates $\mathrm{S}$ protein priming and initiates viral entry is the Type II transmembrane serine protease, TMPRSS2 [20]. Besides TMPRSS2, endosomal protease cathepsin-L (CTS-L) also enhances SARS-CoV-2 entry into host alveolar cells and cardiomyocytes [20]. This observation was supported by the fact that, cathepsin $\mathrm{B} / \mathrm{L}$ involved in vascular remodeling and cardiovascular diseases [21].

\section{Discussion}

For this study, we searched Pubmed, Medline, Embase databases, preprint reports on bioRxiv or medRxiv up to 30 September 2020 utilizing combinations of word variants for coronavirus or nCOV-19, immune signatures, cardiovascular and respiratory system, severe acute respiratory syndrome or SARS-COV-2. In the last century, the world population has witnessed several assaults from RNA viruses, causing millions of deaths. The $21^{\text {st }}$ century is no longer an exception, with the trend continued with significant fear of SARS-COV first appeared in 2002 and rapidly spread to 32 countries and regions. Subsequently the world experienced the outbreak of novel influenza virus in 2009 and MERS-CoV in 2012. In December 2019, a newly emerged pneumoniaassociated respiratory syndrome caused by SARS-CoV-2, spread quickly causing significant human loss globally. Based on the WHO global epidemiological situation report [12], currently the 10 most affected countries in the world are: USA, India, Brazil, Argentina, Spain, France, Russia, Colombia, UK and Peru. The first case of nCOV-19 in US was reported on $1 / 22 / 2020$. Since then, the country has reported $\sim 71.91$ lakh cases, and 205,998 deaths, where as in India, the first case of nCOV-19 in was reported on $1 / 30 / 2020$. Since then, the country has reported $\sim 62.25$ lakh cases, and 97,497 deaths.

\section{Impact of SARS-CoV-2 on immune signatures}

SARS-CoV-2 infection does not go unnoticed by the immune system. IgM is the first type of antibody appeared in the humoral immune response when a person is infected by SARS-CoV-2. Seropositive of IgM indicates a recent infection so it can be used for early diagnosis of acute phase infections with SARS-CoV-2 [22].Within days to weeks, the shortlived, low-affinity IgM antibodies are converted to long-lived, highaffinity $\operatorname{IgG}$ and $\operatorname{Ig} \mathrm{A}$ antibodies. $\mathrm{IgG}$ antibodies are especially high in blood, whereas IgA antibodies are high at mucosal surfaces and in secretions. A study by Guo et al [23] involving 208 nCOV-19 patients revealed $\operatorname{IgM}$ and $\operatorname{IgA}$ antibodies first detected within 3-6 days whereas IgG antibodies detected 10-18 days after symptom onset.

ACE2 expressed in the lung besides heart, kidney, immune cells and intestine [24]. ACE2 is a potential target for SARS-CoV-2 infection, and increased ACE2 expression induced by angiotensin converting enzyme inhibitors (ACEI) / angiotensin-receptor blockers (ARB) would aggravate lung injury in SARS-CoV-2 infected patients [25]. Similar to MERS-CoV and SARS-CoV, the first immune signatures potentially infected by SARS-CoV-2 are type II pneumocytes [19]. Helper T cells are important in the adaptive immune response because they help activate B cells, phagocytes, and killer T cells to target or kill infected cells. Few studies showed that patients who had recovered from SARS-CoV-2 infection carried helper $T$ cells that recognized the $S$ protein $[26,27]$. Diao et al [28] reported that total numbers of T-cells $\left(\mathrm{CD}^{+}{ }^{+} \mathrm{T}\right.$-cells and $\mathrm{CD}^{+}{ }^{+} \mathrm{T}-$ cells) are decreased in patients with SARS-CoV-2 infection, and the surviving T-cells are functionally deranged, suggested an altered function of immune signatures in these patients. Thus, immune cells are not only the entry sites for SARS-CoV-2 infection, but they also possess the cellular machinery to mount an inflammatory response toward an infection. ACE2 is a potential target for SARS-CoV-2 infection, and increased ACE2 expression induced by angiotensin converting enzyme inhibitors (ACEI) / angiotensin-receptor blockers (ARB) would aggravate lung injury of patients with SARS-CoV-2 infection.

Furthermore, certain immune related genes such as human leukocyte antigen (HLA) involved in recognizing pathogens, vary from person to person. The individual HLA, haplotype, and genotype variability likely influence how well the immune system recognizes SARS-CoV-2 infection. Poor recognition of immune system could cause a person to be 
more vulnerable to the virus [29]. Following the development of a vaccine against SARS-Cov-2, individuals with high-risk HLA types may be prioritized for vaccination. It is interesting to study how the variation in HLA affects patients susceptible to SARS-Cov-2 infection. Probably, individual genetic variation may explain differences in the strength of immune responses. This could help identify individuals at higher risk from the SARS-Cov-2 infection.

\section{Cytokine Storm}

Upon entry into alveolar epithelial cells, SARS-CoV-2 replicates rapidly and triggers a strong immune response, resulting in cytokine storm [16]. Immune cells then kill-off the infected alveolar cells in a bid to save them. But during a runaway SARS-CoV-2 infection, when the immune system dumps pro-inflammatory cytokines in excess (hypercytokinaemia) into the lungs, this culling becomes a free-for-all, instead of shooting at a target with a gun, cytokines act as missile launcher. That's where the problem arises. When working properly, inflammatory process is tightly regulated and confined only to infected areas. But sometimes the immune system goes haywire and those cells kill anything in their way, including healthy cells.

Similar to the inflammatory cytokine profile in SARS-CoV and MERS$\mathrm{CoV}$, patients with SARS-CoV-2 also have increased plasma levels of TNF- $\alpha$ [30, 31]. Such an uncontrolled production of inflammatory cytokine is accompanied by infiltration of inflammatory monocytes/macrophages (IMM) into the lung that weakens blood vessels and causes fluid to seep through to the air sacs. From there things can take a sharp turn for the worse. The storm spills into circulatory system and creates systemic issues across multiple organs leading to the accumulation of excessive numbers of immune cells at the lung epithelium. In some of the most severe SARS-CoV-2 infected patients, the cytokine storm, combined with a diminished capacity to pump oxygen to the rest of the body, can result in pulmonary edema, acute cardiac, renal or hepatic injury and death [32]. These studies were supported by a recent study from Ziegler group [24], wherein the authors reported that ACE2 receptors are induced by interferon- $\gamma$, thereby amplifying the infectious cycle in the lungs.

\section{Cardiac manifestation of SARS-CoV-2}

Evidence about the effects of SARS-CoV-2 infection on the cardiovascular system is rapidly growing. The interaction between the viral spike (S) protein and ACE-2, which triggers entry of the virus into host cells, is likely to be involved in the cardiovascular manifestations of nCOV-19, and $20 \%$ of all infection-associated deaths are from cardiac complications. Following SARS-CoV-2 infection, activated cardiomyocyte resident immune signatures sense invading viral pathogens/antigens. At the site of infection, activated virus-specific cytotoxic T lymphocytes-TCLs (e.g. CD8+ T-cell) produce chemokines and antiviral cytokines (e.g. IFN- $\gamma$ and TNF- $\alpha$ ). These cytokines inhibit viral replication and enhance antigen presentation and kill infected cells, while chemokines recruit more innate immune cells to control pathogen burden (Fig.2). Huang et al [30] high-lighted that in patients with SARSCov-2 infection, the imbalance of Th-1 and -2 responses resulted in a cytokine storm. The release of inflammatory cytokines after infection may cause various cardiac manifestation, which include reduction in coronary blood flow, decreases in oxygen supply, destabilization of coronary plaque, and micro thrombogenesis, cardiogenic shock, STelevation myocardial injury, viral myocarditis, lymphopaenia [30], arrhythmia-induced cardiomyopathy and venous thrombosis [33-35].

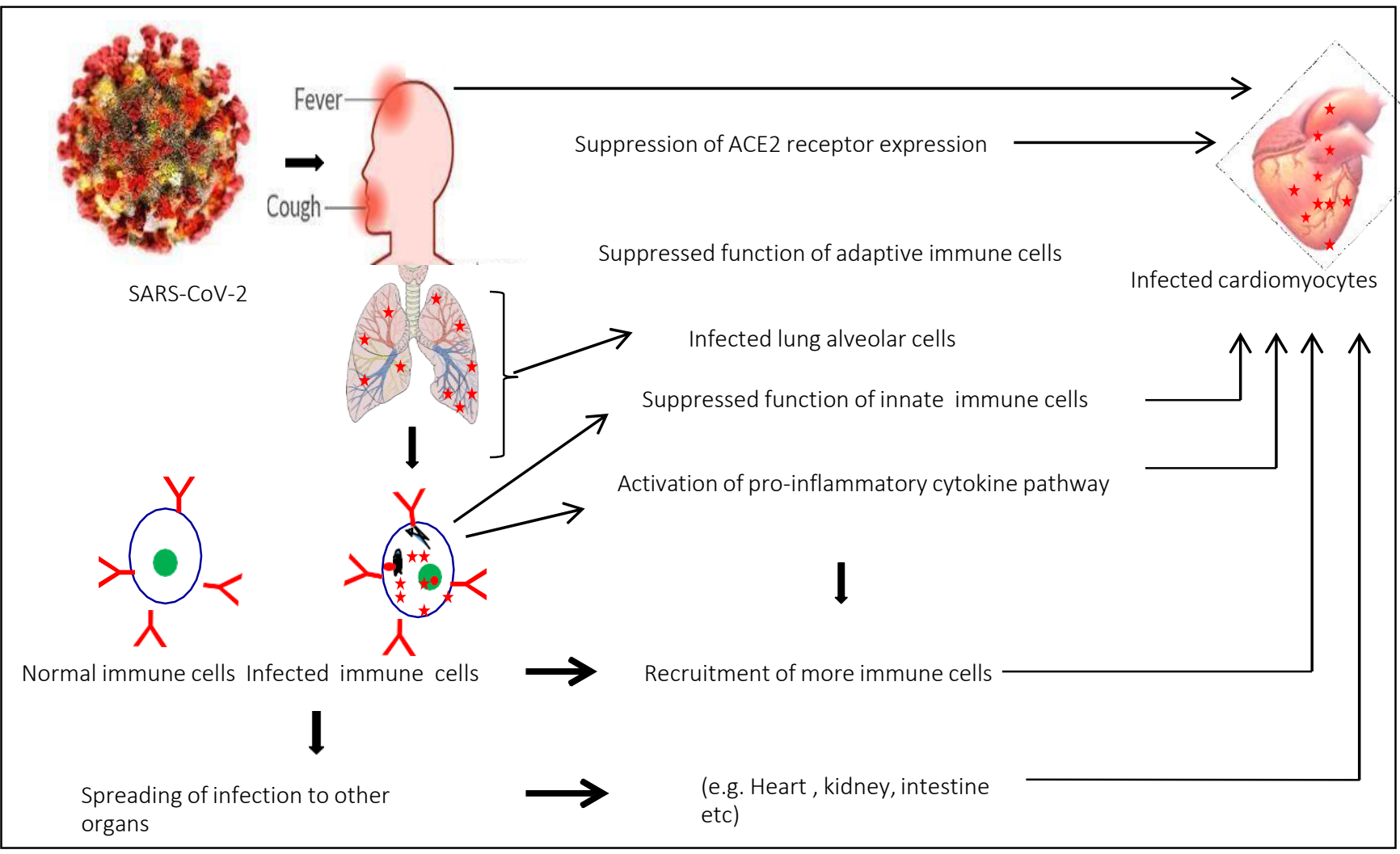

Fig.2. Possible mechanism involved in the pathogenesis of SARS-CoV-2 infection. 
Initiation of immune response against SARS-CoV-2 begins with direct infection of lung alveolar epithelium. Following infection, lung resident immune cells acquire the invading pathogen or antigens from infected alveolar epithelial cells which enter into circulation and adversely influence heart function. The activated virus-specific effector $\mathrm{T}$ cells produce antiviral cytokines and chemokines. Cytokines regulate viral replication and enhance antigen presentation and kill infected alveolar cells. The chemokines produced by activated $\mathrm{T}$ cells recruit more immune cells to control pathogen load.

Patients with underlying cardiovascular comorbidities are more likely to suffer severe cardiac symptoms, which is associated with high mortality from SARS-CoV-2 infection. Ruan et al [36] claimed that total number of patients who died due to myocardial ischemia or heart failure (HF) with or without respiratory failure consists of $40 \%$ of all deaths. Another study indicated that the mortality rate in SARS-CoV-2 infected patients complicated with acute HF is considerably higher as compared to other risk factors, such as elderly, diabetes, chronic pulmonary diseases (CPD) and history of CVD [37]. In a study by Guo et al [23] revealed over 33\% of nCOV-19 patients in the ICU have experience cardiomyopathy.

\section{Role of SARS-CoV-2 associated molecules in cardio- pulmonary signatures}

The range of human cells and tissues targeted by SARS-CoV-2, its potential receptors and associated regulating factors are still largely unknown. Cardiac ACE2 expression was strongest in pericytes and cardiomyocytes. The expression of the proteases encoded by TMPRSS2 and CTSL, which facilitate membrane fusion and viral uptake during SARS-CoV-2 infection, was very high in cardiomyocytes. The $\mathrm{S}$ protein of SARS-CoV-2 undergoes cleavage by a cell surface serine protease, TMPRSS2 [19]. Whether ACE2 and TMPRSS2 are required on the same cell to activate SARS-CoV-2 S protein to invade ACE2 expressing alveolar or cardiomyocytes is yet to be elucidated. However, as active $\mathrm{S}$ protein has a finite lifetime [38], its priming at alveolar or cardiomyocytes will make it effective for viral entry. Thus, we believe that for SARSCoV-2, the ACE2 and TMPRSS2 co-expressing cells may have the highest infectivity.

Beyond the canonical ACE2 and TMPRSS2 based entry, another receptor-CD147 (also known as basigin; encoded by BSG) has been shown to act as a receptor for SARS-CoV-2 in T cell lines and in cell lines of epithelial origin [39]. Presently, the mechanism by which BSG/CD147 mediates non-canonical mode of viral entry in alveolar cells or cardiomyocytes is unknown. In other cells, BSG/CD147 promotes entry of viruses by endocytosis [40]. It is possible that the same mechanism may be operative in the case of SARS-CoV-2.

Once the virus binds to its receptors on host alveolar cells or cardiomyocytes and gains entry, it utilizes a plethora of host genes for its replication. Post replication, most enveloped viruses complete their life-cycle by forming vesicles that bud from the plasma membrane via the cellular endosomal sorting complexes required for transport (ESCRT) machinery. It will be essential to determine how SARS-CoV-2 infection alters the temporal dynamics of host responses cardiomyocyte or alveolar resolution. Such studies would aid in developing rational strategies for management of SARS-CoV-2 infection.

\section{Vaccines}

Currently worldwide, there are several ongoing efforts for vaccine development against SARS-CoV-2 infection. WHO says 172 countries engaging with global vaccine plan against SARS-CoV-2 As per the WHO's latest draft landscape, over 110 vaccines are in different stages of development, and a dozen candidate vaccines for SARS-CoV-2 treatment are in the preclinical/clinical evaluation stage. Phase-I nCOV19 vaccine, Moderna's messenger RNA (mRNA) candidate (mRNA-127) induced rapid and strong immune responses against SARS-CoV-2 in patients older than $55 \mathrm{yrs}$. It induces $\mathrm{CD}^{+}{ }^{+} \mathrm{T}$-cells of the Th-1 phenotype 14 days after the second dose was given. Moderna is also studying mRNA-1273 in a NIAID-led Phase II trial (NCT04283461) designed to assess the safety, reactogenicity, and immunogenicity. The other closely watched vaccine, Oxford-AstraZeneca (SII-ChAdOx1 nCoV-19) prevents SARS-CoV-2 pneumonia. Russian President Vladimir Putin on 11 August 2020 announced that vaccine, Sputnik-V developed by Moscow-based Gamaleya Research Institute of Epidemiology and Microbiology had been registered as world first vaccine against nCOV19 pandemic. Sinovac Biotech Ltd coronavirus vaccine candidate, CoronaVac was approved in July 2020 for emergency use as part of a programme in China to vaccinate high-risk groups. In real situation like this, developing a safe and effective vaccine against SARS-CoV-2 infection is still months away to become available over the counter for public use.

\section{Conclusion and future prospective}

The COVID-19 pandemic, which has exhausted the momentum in China, is still in the exponential phase in the rest of the world. There is still much is unknown about nCoV-19 pandemic including what actually happens to the body when infected by this virus, and how long it can last for any period of time outside of the host. Till date, the association between SARS-CoV-2 infection and its progression has not been confirmed by animal experiments. To meet the urgent need for effective treatment and preventative strategies, global co-ordinate action by researchers is essential to deal health challenges the $\mathrm{nCoV}-19$ pandemic poses to people. It is also important to share information on health technology developments vital to treat $\mathrm{nCoV}-19$ patients. Lastly, strict regulations against the wildlife domestication and consumption should be implemented.

\section{Ethics approval and consent to participate}

Not applicable

Declaration of Conflict of Interest: The author declares no conflict of interest.

\section{Bibliography}

1. Yang D, Leibowitz JL (2015). The structure and functions of coronavirus genomic 3' and 5' ends. Virus Res. 206: 120-133.

2. $\mathrm{Gu} \mathrm{J}$, Korteweg C (2007). Pathology and Pathogenesis of Severe Acute Respiratory Syndrome. Ame J Pathol. 170: 1136-1147.

3. Drosten C, Günther S, Preiser W, van der Werf S, Brodt HR, et al (2003). Identification of a novel coronavirus in patients with severe acute respiratory syndrome. New Engl J Med. 348: 1967-1976.

4. Zaki AM, van Boheemen S, Bestebroer TM, Osterhaus AD, Fouchier RA (2012). Isolation of a Novel Coronavirus from a Man with Pneumonia in Saudi Arabia. New Engl J Med. 367: 1814-1820.

5. Su S, Wong G, Liu Y, Gao GF, Shoujun Li S, et al (2015). MERS in South Korea and China: a potential outbreak threat? Lancet. 385: 2349-2350.

6. Song Z, Xu Y, Bao L, Zhang L, Yu P, et al (2019). From SARS to MERS, thrusting coronaviruses into the spotlight. Viruses. 11:59, doi: 10.3390/v11010059.

7. Fan Y, Zhao K, Shi ZL, Zhou P (2019). Bat Coronaviruses in China. Viruses. 11 (3): 210-43.

8. Hu B, Zeng LP, Yang XL, Yi Ge XY, Zhang W, et al (2017). Discovery of a rich gene pool of bat SARS-related coronaviruses provides new insights into the origin of SARS 
coronavirus. PLoS pathogens. 13: e1006698. doi: 10.1371/journal.ppat.1006698.

9. Guan Y, Zheng BJ, He YO, Liu XL, Zhuang ZX, Cheung CL, et al ( 2003). Isolation and Characterization of Viruses Related to the SARS Coronavirus from animals in southern China. Science. 302(5643): 276-278.

10. Lu G, Wang Q, Gao GF (2015). Bat-to-human: spike features determining 'host jump' of coronaviruses SARS-CoV, MERSCoV and beyond. Trends Microbiol. 23: 468-478.

11. Gorbalenya AE, Baker SC, Baric RS, de Groot RJ, Drosten C, et al ( 2020). Severe acute respiratory syndrome-related coronavirus: the species and its viruses-a statement of the coronavirus study group. bioRxiv. [Preprint], DOI: 10.1038/s41564-020-0695-z.

12. Wang D, Hu B, Hu C, Zhu F, Liu X, (2020a). Clinical Characteristics of 138 Hospitalized Patients with 2019 Novel Coronavirus-Infected Pneumonia in Wuhan, China. JAMA. 323:1061-169.

13. Sexton NR, Smith EC, Blanc H, Vignuzzi M, Peersen OB, et al (2016). Homology-based identification of a mutation in the coronavirus RNA-dependent RNA polymerase that confers resistance to multiple mutagens. J Virol. 90: 74157228.

14. Lu R, Zhao X, Li J, Niu P, Yang B, Wu H, et al (2020) . Genomic characterization and epidemiology of 2019 novel coronavirus: implications for virus origins and receptor binding. Lancet. 395(10224): 565-574.

15. Xu X, Chen P, Wang J, Feng J, Zhou H, et al (2020). Evolution of the novel coronavirus from the ongoing Wuhan outbreak and modeling of its spike protein for risk of human transmission. Sci China Life Sci. 63: 457-460.

16. Zhou P, Yang XL, Wang XG, Hu B, Zhang L, et al (2020) . A pneumonia outbreak associated with a new coronavirus of probable bat origin. Nature. 579 (7798): 270-273.

17. Wu K, Chen L, Peng G, Zhou W, Pennell CA, et al (2020). A Virus-Binding Hot Spot on Human Angiotensin-Converting Enzyme 2 Is Critical for Binding of Two Different Coronaviruses. J Virol. 85: 5331-5337.

18. Li X, Zai J, Zhao Q, Nie Q, Li Y, et al (2020) . Evolutionary history, potential intermediate animal host, and cross-species analyses of SARS-CoV-2. J Med Virol. DOI: 10.1002/jmv. 25731 .

19. Hoffmann M, Weber H, Schroeder S, Nadine Kruger N, Herrler T, et al (2020) . SARS-CoV-2 Cell Entry Depends on ACE2 and TMPRSS2 and Is Blocked by a Clinically Proven Protease Inhibitor. Cell. 181:271-280.

20. Liu CL, Guo J, Zhang X, Sukhova GK, Libby P, et al (2018) . Cysteine protease cathepsins in cardiovascular disease: from basic research to clinical trials. Nature Reviews Cardiology. 15: 351-370.

21. Schroeder H, Cavacini L (2010). Structure and Function of Immunoglobulins. J Allergy Clin Immunol. 125: S41-S52.

22. Guo L, Ren L, Yang S, Xiao M, Chang D, et al (2020). Profiling Early Humoral Response to Diagnose Novel Coronavirus Disease (COVID-19). Clin Infect Dis. 71 (15): 778-785.

23. Ziegler C, Allon SJ, Nyquist SK, Mbano I, Miao VN, et al (2020 ). SARS-CoV 2 Receptor ACE2 is an interferonstimulated gene in human airway epithelial cells and is enriched in specific cell subsets across tissues. SSRN Electron J. 181: 1016.e19-1035.e19.
Kai H, Kai M (2020). Interactions of coronaviruses with ACE2, angiotensin II, and RASinhibitors-lessons from available evidence and insights into COVID-19. Hypertension Research. doi.org/10.1038/s41440-020-0455-8.

25. Grifoni A, Weiskopf D, Ramirez SI, Mateus J, Dan JM, et al (2020). Targets of $\mathrm{T}$ cell responses to SARS-CoV-2 coronavirus in humans with COVID-19 disease and unexposed individuals. Cell. 181 (7): 1489-1501.

26. Braun J, Loyal L, Frentsch M, Wendisch D, Georg P, et al (2020). Presence of SARS-CoV-2 reactive T cells in COVID19 patients and healthy donors. medRxiv [Preprint], Available from: medRxiv 2020.04.17.20061440; doi: DOI: 10.1101/ 2020.04.17.20061440.

27. Diao B, Wang C, Tan Y, Chen X,Liu Y, et al (2020) . Reduction and functional exhaustion of $\mathrm{T}$ cells in patients with coronavirus disease (COVID-19). medRxiv [Preprint], DOI: 10.1101/2020.02.18.20024364.

28. Nguyen A, David JK, Maden SK, Wood MA, Weeder BR, et al (2020). Human leukocyte antigen susceptibility map for SARS-CoV-2. J Virology. 94 (13): e00510-20.

29. Huang Y, Yang C, Xu XF, Xu W, Liu SW (2020). Structural and functional properties of SARS-CoV-2 spike protein: potential antivirus drug development for COVID-19. Acta pharmacologica sinica. 41: 1141-49. J Transl Med 18, 165 (2020)

30. Buonaguro FM, Puzanov I, Ascierto PA (2020). Anti-IL6R role in treatment of COVID-19-related ARDS. J Transl Med. 18: 165, DOI: 10.1186/s12967-020-02333-9.

31. Liu L, Wei Q, Lin Q, Fang J, Wang H , et al (2020). Antispike IgG causes severe acute lung injury by skewing macrophage responses during acute SARS-CoV infection. JCI Insigh. 4: e123158.

32. Fried JA, Ramasubbu K, Bhatt R, Topkara VK, Clerkin KJ, et al (2020). The variety of cardiovascular presentations of COVID-19. Circulation. 141:1930-1936

33. Juusela A, Nazir M, Gimovsky M (2020). Two Cases of COVID-19 Related Cardiomyopathy in Pregnancy. American J Obstet Gynecol. 2(2): DOI: 10.1016/j.ajogmf.2020.100113.

34. Dabbagh MF, Aurora L, D'Souza P, Weinmann AJ, Bhargava P, et al (2020). Cardiac Tamponade Secondary to COVID-19. JACC Case Reports.2 (9): 1326-1330

35. Ruan Q, Yang K, Wang W, Jiang L, Song J (2020). Clinical predictors of mortality due to COVID-19 based on an analysis of data of 150 patients from Wuhan, China. Intensive care medicine. 46(5): 846-848.

36. Mahdavi FS, Khavandegar A, Mardi P (2020). Impact of SARS-CoV-2 On St-Elevation Myocardial Infarction (STEMI). Ame J Biomed Res. 73-75, MS.ID.001355.

37. Shulla A, Heald-Sargent T, Subramanya G, ZhaoJ, PerlmanS., et al. (2011) A transmembrane serine protease is linked to the severe acute respiratory syndrome coronavirus receptor and activates virus entry. J Virol. 85: 873-882.

38. Wang K, Chen W, Zhou YS, Lian JQ, Zhang Z, et al (2020b). SARS-CoV-2 invades host cells via a novel route: CD147-spike protein. bioRxiv. [Preprint], 2003.2014.988345.

39. Pushkarsky T, Zybarth G, DubrovskyL, YurchenkoV, Tang H, et al (2001). CD147 facilitates HIV-1 infection by interacting with virus-associated cyclophilin A. Proc Natl Acad Sci USA. 98:6360-6365.

24. 